\title{
Near-Infrared Fluorescence from Nanodiamond for Multimodal Bioimaging
}

\author{
DOI: $10.17691 / \mathrm{stm} 2018.10 .1 .06$
}

Received November 2, 2017

Y.-C. Lin, PhD, Research Fellow, Department of Physics'; Research Fellow²;

L.-W. Tsai, Master Student, Department of Physics";

E. Perevedentseva, PhD, Research Fellow, Department of Physics'; Research Fellow, S.I. Vavilov Department of Luminescence ${ }^{3}$;

A. Karmenyan, PhD, Research Fellow, Department of Physics";

C.-L. Cheng, PhD, Professor, Department of Physics ${ }^{1}$

${ }^{1}$ National Dong Hwa University, 1, Sec. 2, Da-Hsueh Rd., Shoufeng, Hualien, 97401, Taiwan, R.O.C.,

${ }^{2}$ Institute of Physics, Academia Sinica, 128, Sec. 2, Academia Rd., Nangang Dist., Taipei, 11529, Taiwan, R.O.C.,

${ }^{3}$ P.N. Lebedev Physical Institute, Russian Academy of Sciences, 53 Leninskiy Prospekt, Moscow, 119991,

Russia

Nanodiamonds (ND) are emerging as a promising candidate for the multimodal bioimaging due to their optical and spectroscopic properties. Fluorescence properties of ND are determined by defects and admixtures in the crystal lattice. The most developed bioapplications of the ND fluorescence are using nitrogen-vacancy centers. However they emit fluorescence in the visible region which overlaps with the autofluorescence from biological objects.

The aim of the study was to analyze the fluorescence of nickel-related color center in nanodiamond with emission in the near-infrared range $(883-885 \mathrm{~nm})$ in terms of its applications for bioimaging using one-photon and two-photon excitations.

Materials and Methods. Synthetic diamond powders (Kay Diamond, USA) of sizes in the range from $100 \mathrm{~nm}$ to $2.5 \mu \mathrm{m}$ were carboxylated and characterized with Raman and photoluminescence spectroscopy at one-photon and two-photon excitation. Baby hamster kidney cells were treated with $500 \mathrm{~nm} \mathrm{ND}$ for $8 \mathrm{~h}$ and subjected to microscopic investigations using laser confocal fluorescence scanning microscopy and photoluminescence mapping.

Results. The effects of the particles size, temperature and excitation conditions on the fluorescence of Ni-related center are studied. Variability of the emission with sizes (as well as with excitation wavelength and temperature) gives the possibility to select the most suitable nano- or microparticles to use as a fluorescent probe. The two-photon excitation of Ni centers in nano- and microdiamond are demonstrated. The possibility to use Ni color center for bioimaging is presented using confocal fluorescence imaging and fluorescence mapping of distribution of $500 \mathrm{~nm}$ ND in baby hamster kidney cells. The emission of Ni-related center ( $885 \mathrm{~nm}$ ) showing no photobleaching and no damage to the baby hamster kidney cells and the location of ND is clearly observed relatively the cells.

Conclusion. Fluorescence from Ni-related color center at one-photon and two-photon excitation can be an option in biological imaging to avoid cell autofluorescence and to shift the excitation to lower energy laser excitation which is safer and transparent for biological objects.

Key words: nanodiamond; Ni-related defects; near-infrared emission; color center; fluorescence; bioimaging.

How to cite: Lin Y.-C., Tsai L.-W., Perevedentseva E., Karmenyan A., Cheng C.-L. Near-infrared fluorescence from nanodiamond for multimodal bioimaging. Sovremennye tehnologii v medicine 2018; 10(1): 49, https://doi.org/10.17691/stm2018.10.1.06

\section{Introduction}

Nanodiamond (ND) has been identified as a promising candidate for the multifunctional applications in biomedical researches for its physical/chemical properties and numerous confirmed biocompatible studies. Particularly, optical and spectroscopic properties of ND are considered for the purposes of multimodal bioimaging (including drug delivery tracing) and biosensing [1, 2].

The defect-originated color centers in ND are suitable for numerous possible applications ranging from quantum information processing as single-photon sources [3-5] to optical markers for cellular imaging [1, $2,6]$. The most well-studied and utilized defect to date is nitrogen-vacancy (NV) color centers which appear in neutral $\left(\mathrm{NV}^{\circ}\right)$ and negative $\left(\mathrm{NV}^{-}\right)$charge states with zerophonon lines (ZPL) at $575 \mathrm{~nm}(2.156 \mathrm{eV})$ and $637 \mathrm{~nm}$ $(1.945 \mathrm{eV})$, respectively $[3,5,7]$. NV color centers emit stable emission with broad spectral ranges at room temperature and are recently used for bioimaging and biosensing [8]. However, the limitation is the fluorescence wavelength predominantly are between 400 to $550 \mathrm{~nm}$,

Corresponding author: Chia-Liang Cheng, e-mail: clcheng@gms.ndhu.edu.tw 
this overlaps with most biomolecules that absorb light between 280 to $500 \mathrm{~nm}$ ranges [9], some obstacles do exist for bioapplications using NV centers. Therefore, for an improved biolabelling, there is a need the emission be shifted above this wavelength region.

To circumvent the obvious limitation associated with the use of NV centers, the potential use of fluorescence in the near-infrared region from silicon-vacancy (with ZPL at $737 \mathrm{~nm}$ ) [6] or some of nickel (Ni) centers [10] would be an alternative for the detection, as its emission is far away from most of the biomolecules and commercial dyes. Synthetic diamond grown by the high-pressure hightemperature technique uses a nickel-containing metallic catalyst, and nickel is usually inevitably included in the resulting diamond. Particularly, dispersed nickel atoms can be incorporated in diamond producing color centers with emission in the infrared region, result in the $1.4 \mathrm{eV}$ Ni-related center (with ZPL near $885 \mathrm{~nm}$ ) with negative nickel ion in the center of a diamond divacancy, and nickel-nitrogen complex $\mathrm{NE}^{8}$ with $\mathrm{ZPL}$ at $796 \mathrm{~nm}$ formed by a substitutional nickel atom with four adjacent nitrogen atoms using chemical vapor deposition (CVD) method. It is also possible to fabricate single nickel-nitrogen defects in diamond [11]. Recently, the near-infrared emitting Nirelated centers are produced in ND [11, 12]. Ni-related optical centers are considered as alternatives to the NV. A number of schemes are available for creating Nirelated optical centers in diamond, such as incorporation of $\mathrm{Ni}$ during CVD growth or by ion implantation. Ion implantation promises the best control over the location, density and proximity to the surface of the optical center [13]. Note that Ni centers from Ni-enriched ND can be excited with different wavelength, including wavelength in the red region [11, 12] and that increases their interest for bioimaging. Additional imaging opportunity is opened due to the observed two-photon excitation of ND demonstrated previously for NV centers [14]. It allows shifting the excitation to the infrared region for deeper penetration of the excitation light into the tissue, increasing the spatial resolution and the laser penetration depth. Also it decreases the out of focus effects on the bio-object and results in decreased photobleaching and photodamage of the sample [15].

In this report we discuss the Ni-related $(1.4 \mathrm{eV})$ center observed in the as-received ND $[16,17]$ excited with ultraviolet light and with two-photon femtosecond excitation in infrared range with fluorescence detected in the near-infrared range (with peak near $885 \mathrm{~nm}$ ). The effects of ND size, temperature and excitation conditions on the emission are discussed. The application of $\mathrm{Ni}$ center as a biolabel using fluorescence mapping technique is demonstrated. The possibility to use this color center for bioimaging applications is presented using fluorescence mapping of distribution of $500 \mathrm{~nm}$ ND in baby hamster kidney cells (BHK). The emission of Nirelated center $(885 \mathrm{~nm})$ showing no photobleaching and no damage to the BHK cells and the location of ND is clearly observed relatively the cells.

\section{Materials and Methods}

In this study, synthetic ND powders (Kay Diamond, USA) of sizes in the range from $100 \mathrm{~nm}$ to $2.5 \mu \mathrm{m}$ were used. The ND powders were carboxylated, resulting in carboxylated ND, using strong acid treatments to remove the metallic impurities, non-diamond carbon and forming carboxyl groups on the surface as reported in detail earlier [18]. For characterizations, the obtained ND powders of various sizes were separately dispersed in de-ionized water in a concentration of $2 \mathrm{mg} / \mathrm{ml}$. From each suspension, $20 \mu \mathrm{l}$ was dropped onto single crystal silicon (100) wafer and dried. Raman spectra and photoluminescence (PL) of ND's were measured using confocal micro-Raman spectrometer (Jobin Yvon, T64000; HORIBA Ltd, Japan) equipped with a liquid $\mathrm{N}_{2}$ cooled charge-coupled device detector at $532 \mathrm{~nm}$ (solid state; Elforlight, UK) and $325 \mathrm{~nm}$ ( $\mathrm{He}-\mathrm{Cd}$ gas phase; Kimmon Koha, Japan) wavelength laser excitations. The laser power through the objective lens was estimated to be $2 \mathrm{~mW}$ (532 nm excitation, measured from objective) and $20 \mathrm{~mW}$ (325 nm excitation, measured from laser's output), which did not raise the sample temperature significantly. For low-temperature measurements, the samples were mounted in a Linkam variable temperature microscope stage (THMS 600; Linkam Scientific, UK) and the temperature was allowed to stabilize for at least $5 \mathrm{~min}$ before acquiring the spectra.

Two-photon excited spectra measurements were measured using Shamrock 303i Spectrograph (Andor Technology Ltd, UK) at femtosecond tunable excitation of Chameleon Ultra II Ti:Sapphire laser (Coherent, USA), excitation wavelength $760 \mathrm{~nm}$, repetition rate $80 \mathrm{MHz}$, pulse duration $140 \mathrm{fs}$, input laser power $96 \mathrm{~mW}$, with band-pass filter FF01-716_43-25. The registration was done in 800-1000 nm spectroscopic range with single photon counting system PicoHarp 300 (PicoQuant, Germany) and cooled photomultiplier tube connected with optical microscope Olympus IX71 (Olympus, Japan), objective magnification was $40 x$.

Baby hamster kidney cells were cultured in complete DMEM (Dulbecco's modification of Eagle's medium) medium with $2 \%$ fetal bovine serum (Invitrogen Co., USA), at $37^{\circ} \mathrm{C}$ and $5 \% \mathrm{CO}_{2}$ in a humidified incubator (310/Thermo; Forma Scientific, Inc., USA). The cells were grown on coverslips in a $22 \mathrm{~mm}$ Petri dish for $16 \mathrm{~h}$ before treatment with ND, and then co-incubated with $500 \mathrm{~nm}$ ND for $8 \mathrm{~h}$. After the treatment, the cells were washed with isotonic PBS (phosphate buffer saline, $\mathrm{pH}$ 7.4) and then fixed with $4 \%$ paraformaldehyde solution in PBS for $1 \mathrm{~h}$ at $37^{\circ} \mathrm{C}$. The samples of cells with ND adhered to the coverslips were used for microscopic investigations. Laser confocal scanning microscope (Leica TCS SP5; Leica Microsystems, Germany) equipped with argon laser (458/476/488/ $514 \mathrm{~nm}$ wavelength) was employed for fluorescence studying of the interactions of ND and BHK.

Photoluminescence mapping was recorded using 
an avalanche photodiodes detector at $325 \mathrm{~nm} \mathrm{He-Cd}$ gas phase laser excitation (Kimmon Koha, Japan). The laser power passed through a $40 \times$ objective lens was measured to be $100 \mu \mathrm{W}$. Near-infrared edge long pass filter (Semrock, Canada) which transmitted wavelength from $800 \mathrm{~nm}$ to $1200 \mathrm{~nm}$ was used. Each figure captured area $30 \times 30 \mu \mathrm{m}$ with $100 \times 100$ pixels resolution.

\section{Result and Discussion}

Figure 1 depicts the photoluminescence spectra from $500 \mathrm{~nm}$ ND measured with $532 \mathrm{~nm}$ (a)-(c) and $325 \mathrm{~nm}$ (d)-(f) wavelength laser excitations. Due to the ND luminescence defect nature and abundant defect centers of ND, varying excitations can excite different color centers. As shown in Figure 1 (a), at $532 \mathrm{~nm}$ wavelength laser excitation the emission from $\mathrm{NV}^{-}$center predominates with the sideband centered near $690 \mathrm{~nm}$ and ZPL at $638 \mathrm{~nm}$. This is more clear and pronounced at low-temperature $(110 \mathrm{~K})$ measurements. In Figure 1 (d) with $325 \mathrm{~nm}$ wavelength laser excitation the luminescence is mostly in the 470-600 nm range, where $\mathrm{N}^{3}, \mathrm{H}^{4}, \mathrm{NV}^{0}$ centers emit $[19,20]$; the shoulder at higher wavelengths of this peak can be attributed to the emission from $\mathrm{NV}^{0}$ center (ZPL at $575 \mathrm{~nm}$ isn't visible here) and the sideband near $600 \mathrm{~nm}$.

At ultraviolet excitation, an isolated peak at $885 \mathrm{~nm}$ is observed, both at room temperature and at $110 \mathrm{~K}$ (Figure $1(\mathrm{e})$ ), while at $532 \mathrm{~nm}$ wavelength laser excitation this weak peak in this range is observed only at low temperature (Figure 1 (b)). The peak at $885 \mathrm{~nm}$ is attributed to $\mathrm{Ni}$-related center with $\mathrm{Ni}^{+}$ion in the center of a diamond divacancy, where $\mathrm{Ni}$ is probably not bonded to the carbon neighbors, described also as $1.4 \mathrm{eV}$ center. For this center emission, the doublet with maximums near 1.401 and $1.404 \mathrm{eV}$ have been previously observed [11], originates from a transition between an excited state and two ground states with the lines splitting, from the $\mathrm{C}$ and $\mathrm{Ni}$ isotopic structure. The exact positions of the 1.4$\mathrm{eV}$ lines depend on the strain in the sample [11]. Despite the variability of some characteristic, the Ni-related

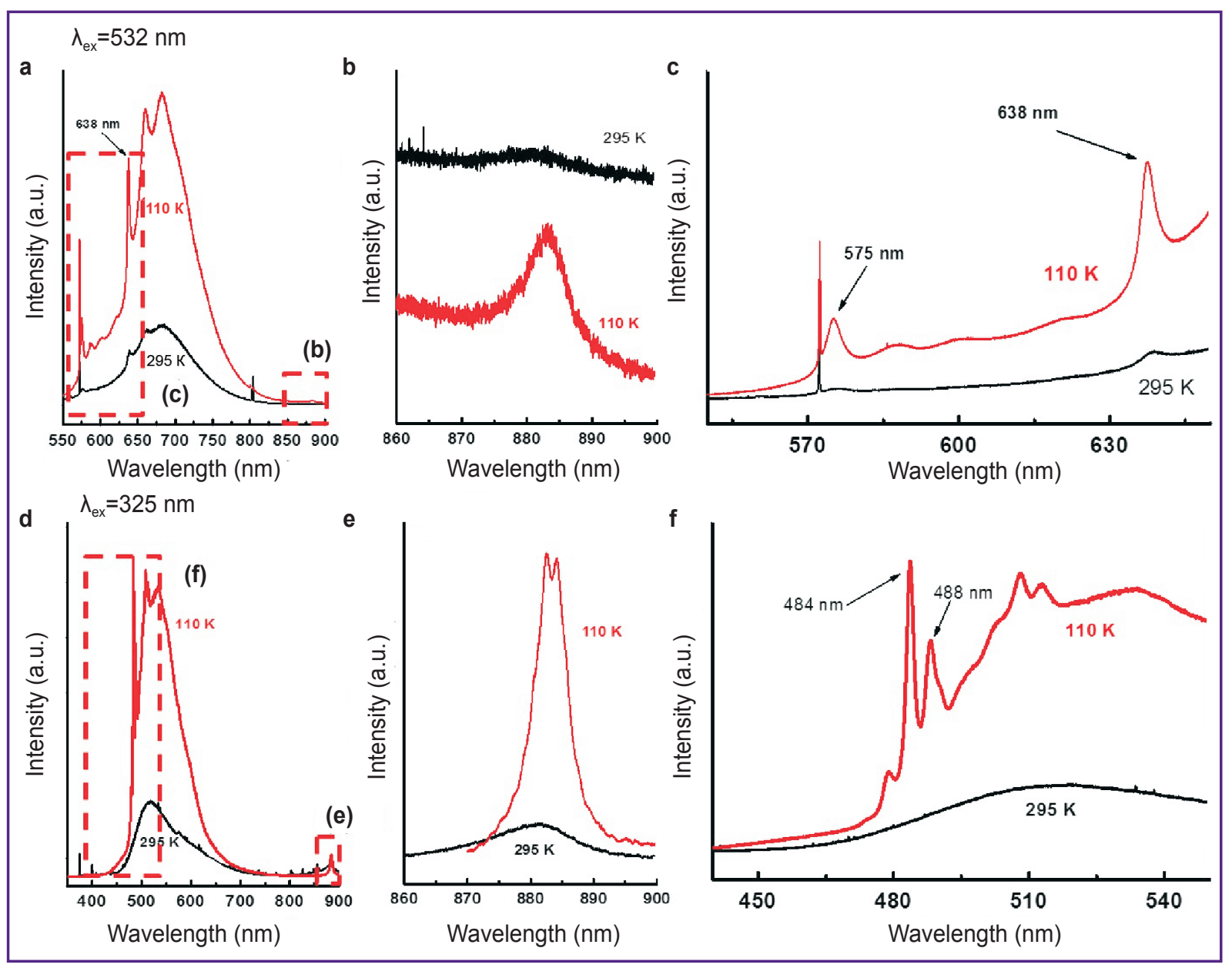

Figure 1. Photoluminescence spectra from $500 \mathrm{~nm}$ nanodiamond measured at (a)-(c) $532 \mathrm{~nm}$ and (d)-(f) $325 \mathrm{~nm}$ wavelength excitations

The weak peak at $880 \mathrm{~nm}$ is expanded in (b) and (e) shown the $1.4 \mathrm{eV} \mathrm{Ni}$ center emission. In (c) and (f), the region near the excitation is magnified revealing details of the fluorescence emission 
centers can be observed for many synthetic and natural diamonds [21, 22] including high-temperature highpressure ND [17, 23].

In addition to the $1.4 \mathrm{eV}$ center in the studied ND, at ultraviolet excitation we can clearly observe doubled peaks at $484 \mathrm{~nm}(2.56 \mathrm{eV})$ and $488 \mathrm{~nm}(2.54 \mathrm{eV})$, considered as also Ni-related centers with $\mathrm{Ni}^{-}$occupies a tetrahedral position, substituting for a carbon atom, and nickel-nitrogen defect $\left(\mathrm{NE}^{2}\right)$, described as $\mathrm{Ni}^{+}$ion bound to divacancy surrounded by two or three nitrogen atoms in nearest coordination sphere, correspondingly [24]. Observed in both natural and synthetic diamond (including ND), the $\mathrm{Ni}$ centers have been studied with PL spectroscopic analysis [23] as well as with electron paramagnetic resonance technique [25] and with analysis of cathodoluminescence [17, 26]. The correlation between $1.4 \mathrm{eV}$ center and $2.56 \mathrm{eV}$ center has been established before [27]. The doublets at 484/488 $\mathrm{nm}$ are although strong enough are excited only at low temperature $(160 \mathrm{~K})$ and in the blue-green range. This may have photonic applications, but may not be suitable for bioimaging purposes. Along with the PL, diamond Raman signal $\left(1332 \mathrm{~cm}^{-1}\right.$, phonon mode of $\mathrm{sp}^{3}$-bound carbon) is observed in the spectra. The narrow peaks appear at $572.5 \mathrm{~nm}$ (at $532 \mathrm{~nm}$ wavelength laser excitation) and near $340 \mathrm{~nm}$ (at $325 \mathrm{~nm}$ wavelength laser excitation), but are not the focus for this study. At room temperature, the doublet $1.401 / 1.404 \mathrm{eV}(885 / 883 \mathrm{~nm})$ is not resolved. With the temperature decreasing to $110 \mathrm{~K}$ (see Figure $1(\mathrm{e})$ ), the peak splits into doublet. Note that we didn't observe the fine structure of the lines, determined by the isotopes (Ni, C) composition [13, 27].

We observed the characteristic emission of ND of different sizes from $100 \mathrm{~nm}$ to $2.5 \mu \mathrm{m}$. In Figure 2 (a) the spectra measured with $325 \mathrm{~nm}$ wavelength laser excitation at room temperature (normalized to the peak height at $500 \mathrm{~nm}$ wavelength) are shown. As seen from the spectra the PL intensity of the $1.4 \mathrm{eV}$ center relative to other color centers is higher when the size of particles is larger. Variability of the emission with ND sizes (as well as with excitation wavelength and temperature) give the possibility to select the most suitable ND to use as a fluorescent probe. In Figure 2 (b) the spectra excited with $325 \mathrm{~nm}$ wavelength laser excitation are compared with the spectra measured with two-photon excitation using a $760 \mathrm{~nm}$ wavelength femtosecond laser. The maximum of emission at 883-885 nm coincides at one- and twophoton excitations for both diamond powders of sizes $500 \mathrm{~nm}$ and $2.5 \mu \mathrm{m}$. However, in the spectrum from $2.5 \mu \mathrm{m}$ microdiamond additional peaks are observed. Interestingly, that their peaks also can be attributed as $\mathrm{Ni}$-containing defects with luminescence maximums in the 845-855 $\mathrm{nm}$ range (or 1.448-1.466 eV, Ni- and O-containing defect) and 996 (1.245 eV) [24]. It has been shown for the first time that two-photon excited luminescence of $\mathrm{Ni}$-containing defects from diamond nano- and microparticles opens opportunities for further development of these particles.

The fluorescence imaging and mapping (distribution of signal at certain wavelength) are widely used for biological cells and tissues visualization. ND has been proposed as an alternative to fluorescence dye due to its biocompatibility, chemical stability, easy surface modification and functionalization, and useful spectroscopic properties [1, 2]. Nitrogen-vacancy centers have been demonstrated to be useful for bioimaging, and the methods to increase the number
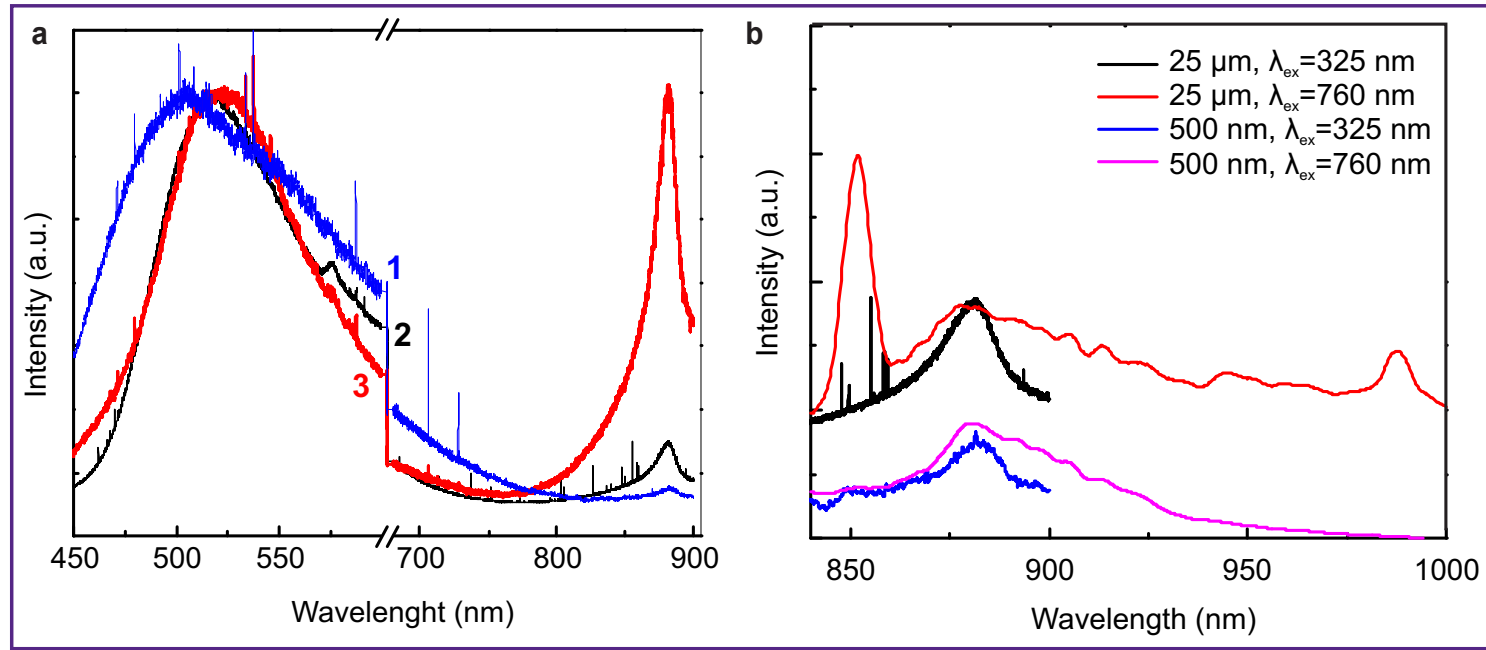

Figure 2. Measurements of photoluminescence spectra

(a) Photoluminescence spectra measured with a $325 \mathrm{~nm}$ wavelength laser excitation at room temperature. The sizes of nanodiamond are (1) $100 \mathrm{~nm}$, (2) $500 \mathrm{~nm}$, and (3) $25 \mu \mathrm{m}$. (b) Photoluminescence spectra measured with a two-photon excitation with femtosecond laser, at $760 \mathrm{~nm}$ wavelength at room temperature from $500 \mathrm{~nm}$ and $25 \mu \mathrm{m}$ diamond particles in comparison with one-photon excitation 


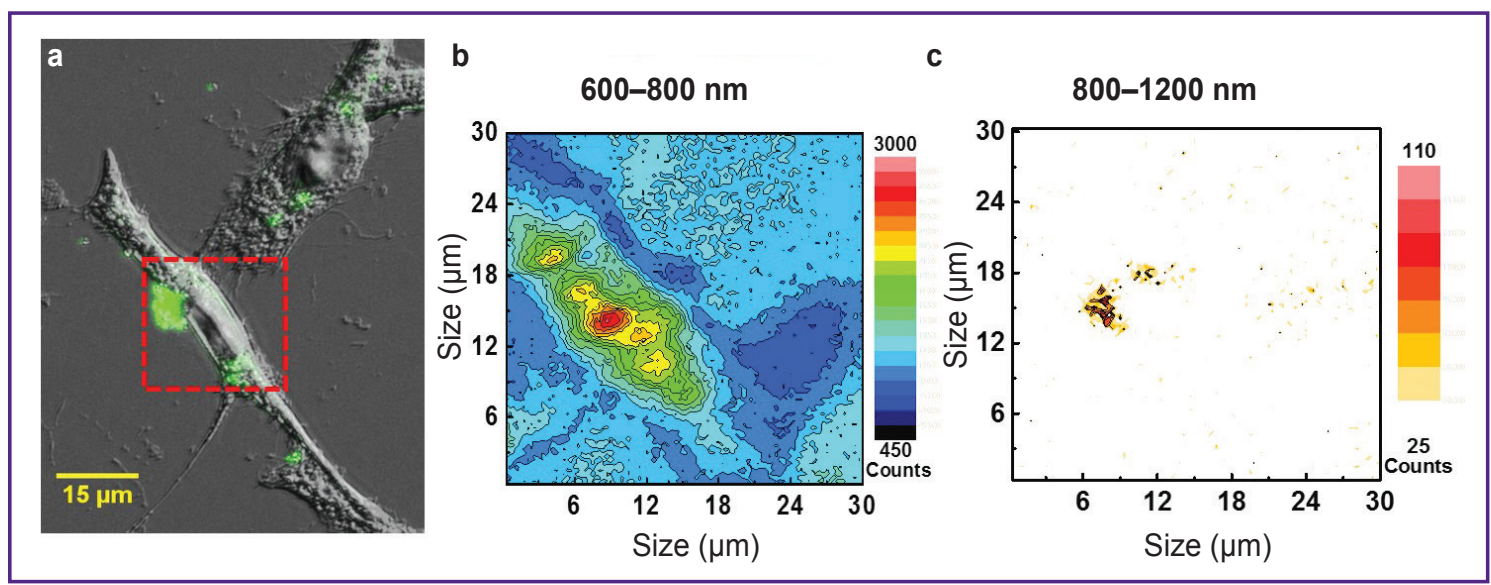

Figure 3. Laser confocal image and photoluminescence mapping of $500 \mathrm{~nm}$ carboxylated nanodiamond with baby hamster kidney cells

(a) Optical image of single cell containing $500 \mathrm{~nm}$ carboxylated nanodiamond excited using $488 \mathrm{~nm}$ wavelength laser. Photoluminescence mapping of $500 \mathrm{~nm}$ carboxylated nanodiamond focused on (b) NV- center (620$640 \mathrm{~nm})$ and (c) Ni-related center $(885 \mathrm{~nm})$ under $325 \mathrm{~nm}$ wavelength laser excitation exposure; each pixel exposure time $0.1 \mathrm{~s}$, total integral time $17 \mathrm{~min}$, scanning area from the red square in (a). Lower photon counts are the cells edges and higher photon counts are the respective centers

of NV centers in ND to enhance the fluorescence have also been developed [9]. However, autofluorescence from biological objects overlaps with the emission of $\mathrm{NV}$ centers $(\sim 500-800 \mathrm{~nm})$; this hinders the observation of using ND for imaging. Using the color centers with emission in red and near-infrared range would be advantageous as alternative to molecular dyes and NV centers fluorescence. In practice, there is indeed the necessity to separate the ND signal from the cell autofluorescence to improve the imaging, delivery tracing, monitoring, and corresponding methods [28]. Imaging using $\mathrm{Ni}$ center (1.4 eV system) fluorescence allows separation from autofluorescence.

We tested the possibility of employing $\mathrm{Ni}$ color center for bioimaging using fluorescence mapping with $500 \mathrm{~nm}$ ND in BHK cells excited with $488 \mathrm{~nm}$ wavelength laser. Figure 3 (a) presents the confocal optical image of BHK cells with a single $500 \mathrm{~nm}$ $\mathrm{ND}$, detected the $\mathrm{N}-\mathrm{V}-\mathrm{N}\left(\mathrm{H}^{3}\right)$ color center emission, and collected in the 495 to $550 \mathrm{~nm}$ wavelength region. Shown in Figure 3 (b), the $\mathrm{NV}^{-}$center fluorescence collected in the $600-800 \mathrm{~nm}$ range. In Figure 3 (c), the Ni-related center $(885 \mathrm{~nm})$ excited with $325 \mathrm{~nm}$ wavelength laser and collected in the $800-1200 \mathrm{~nm}$ region of the red square (see Figure $3(\mathrm{a})$ ) demonstrating the ND's $1.4 \mathrm{eV}$ defect PL is applicable for bioimaging. When comparing the ND image between

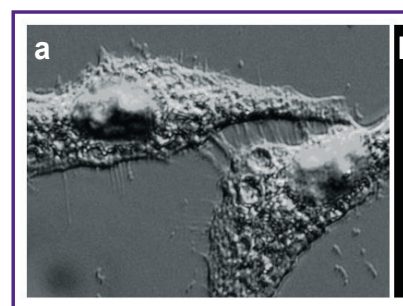

d

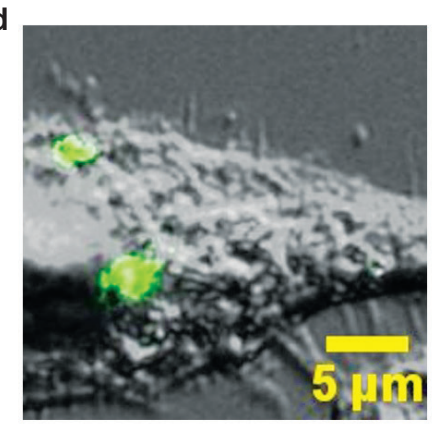

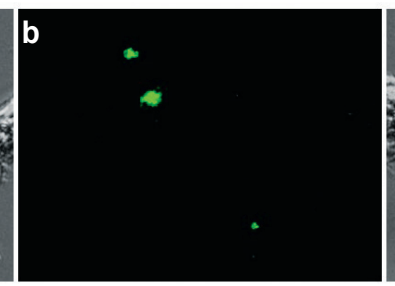

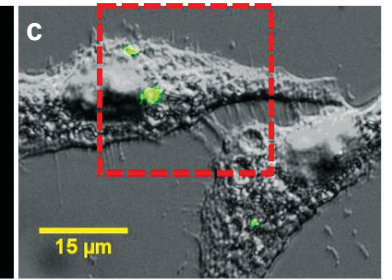

e 30

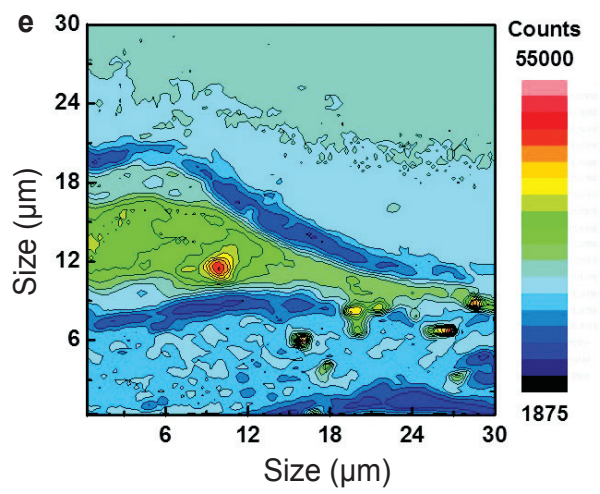

Figure 4. Laser confocal image and photoluminescence mapping of $500 \mathrm{~nm}$ carboxylated nanodiamond with baby hamster kidney cells

(a) Optical image of single cell containing $500 \mathrm{~nm}$ carboxylated nanodiamond excited using a $488 \mathrm{~nm}$ wavelength laser; (b) photoluminescence of $500 \mathrm{~nm}$ carboxylated nanodiamond detects $\mathrm{H}^{3}$ center (495-550 nm); (c) merged images from (a) and (b). (d) Zoom in from the red square in (c). (e) Photoluminescence mapping of Ni-related center $(885 \mathrm{~nm})$ under $325 \mathrm{~nm}$ wavelength laser excitation exposures; each pixel exposure time $0.1 \mathrm{~s}$, total integral time $17 \mathrm{~min}$. Lower photon counts are the cells edges and higher photon counts are the respective centers 
using NV-related center and Ni-related one can easily see the advantage of using the near-infrared emission of the Ni-related centers.

In Figure 4, we present the imaging using the $1.4 \mathrm{eV}$ $\mathrm{Ni}, \mathrm{H}^{3}$, and $\mathrm{NV}$ centers fluorescence. Figure $4(\mathrm{a})$ is the confocal image of BHK cells with $500 \mathrm{~nm}$ ND, the green color shows the $\mathrm{H}^{3}$ color center emission, collected in 495 to $550 \mathrm{~nm}$ wavelength region (Figure 4 (b)) and excited by $488 \mathrm{~nm}$ wavelength laser. The distribution of the PL intensity that detects the $\mathrm{NV}^{-}$defect emission is in the $600-800 \mathrm{~nm}$ wavelength range with $325 \mathrm{~nm}$ wavelength laser excitation, shown in Figure 4 (e). The scanning area is in red square in Figure 4 (c). The combined $1.4 \mathrm{eV}$ defect PL intensity in the 800$1200 \mathrm{~nm}$ wavelength range is mapped in Figure 4 (c). Lower photon counts are the cells edges and higher photon counts are the respective centers, demonstrating the ND's $1.4 \mathrm{eV}$ defect PL is more clear for bioprobing than other defect centers of ND. Figure 4 (d) shows the zoom in from the red square in (c). The PL mapping of Ni-related center $(885 \mathrm{~nm})$ under $325 \mathrm{~nm}$ wavelength laser excitation exposures is displayed in Figure 4 (e). Lower photon counts are the cells edges and higher photon counts are the respective centers. Note that for the bioimaging it represents a major advantage that $\mathrm{Ni}-$ related center fluorescence does not overlap with the cell's autofluorescence.

\section{Conclusion}

In this work, we study the photoluminescence properties of nanodiamond of various sizes at different condition of temperature and excitation laser wavelength, focusing on the use of Ni-related center which the fluorescence is emitted in the $1.401 / 1.404 \mathrm{eV}$ $(885 / 883 \mathrm{~nm})$ region. The PL intensity of the $1.4 \mathrm{eV}$ center relative to other color centers is higher when the size of particles is larger. Variability of the emission with ND sizes (as well as with excitation wavelength and temperature) gives the possibility to select the most suitable ND for use as a fluorescent probe. The two-photon excitation of $\mathrm{Ni}$ centers luminescence in nano- and microdiamond particles is demonstrated. In terms of the ND applications we demonstrate the possibility to use the $\mathrm{Ni}$ color center for bioimaging at ambient conditions applying the ND in baby hamster kidney cells. The emission of Ni-related center $(885 \mathrm{~nm})$ showing no photo-bleaching and no damage to the baby hamster kidney cells and the location of ND is clearly observed relatively the cells as compared to using NV centers imaging. This demonstrates fluorescence from Ni-related color center at one-photon and two-photon excitation can be an alternative option in biological imaging applications to avoid cell autofluorescence and to shift the excitation in range of higher safety and higher transparency for biological objects.

Acknowledgements. The authors would like to thank the Ministry of Science and Technology of Taiwan for financially supporting this research under Contracts No. MOST 106-2112-M-259-009-MY3.

Conflict of interest. The authors have no conflict of interests to disclose.

\section{References}

1. Turcheniuk K., Mochalin V.N. Biomedical applications of nanodiamond (review). Nanotechnology 2017; 28(25): 252001, https://doi.org/10.1088/1361-6528/aa6ae4.

2. Perevedentseva E., Lin Y.C., Jani M., Cheng C.L. Biomedical applications of nanodiamonds in imaging and therapy. Nanomedicine 2013; 8(12): 2041-2060, https://doi. org/10.2217/nnm.13.183.

3. Aharonovich I., Castelletto S., Simpson D.A., Su C.-H., Greentree A.D., Prawer S. Diamond-based single-photon emitters. Rep Prog Phys 2011; 74(7): 076501, https://doi. org/10.1088/0034-4885/74/7/076501.

4. Wrachtrup J., Jelezko F. Processing quantum information in diamond. J Phys Condens Matter 2006; 18(21): S807-S824, https://doi.org/10.1088/0953-8984/18/21/s08.

5. Pezzagna S., Rogalla D., Wildanger D., Meijer J., Zaitsev A. Creation and nature of optical centres in diamond for single-photon emission - overview and critical remarks. New J Phys 2011; 13(3): 035024, https://doi.org/10.1088/13672630/13/3/035024.

6. Vlasov I.I., Shiryaev A.A., Rendler T., Steinert S., Lee S.Y., Antonov D., Vörös M., Jelezko F., Fisenko A.V., Semjonova L.F., Biskupek J., Kaiser U., Lebedev O.I., Sildos I., Hemmer P.R., Konov V.I., Gali A., Wrachtrup J. Molecularsized fluorescent nanodiamonds. Nat Nanotechnol 2014; 9(1): 54-58, https://doi.org/10.1038/nnano.2013.255.

7. Jelezko F., Wrachtrup J. Single defect centres in diamond: a review. Phys Stat Sol A 2006; 203(13): 3207-3225, https://doi.org/10.1002/pssa.200671403.

8. Balasubramanian G., Lazariev A., Arumugam S.R., Duan D.W. Nitrogen-vacancy color center in diamond-emerging nanoscale applications in bioimaging and biosensing. Curr Opin Chem Biol 2014; 20: 69-77, https://doi.org/10.1016/j. cbpa.2014.04.014.

9. Fu C.C., Lee H.Y., Chen K., Lim T.S., Wu H.Y., Lin P.K., Wei P.K., Tsao P.H., Chang H.C., Fann W. Characterization and application of single fluorescent nanodiamonds as cellular biomarkers. Proc Natl Acad Sci USA 2007; 104(3): 727-732, https://doi.org/10.1073/pnas.0605409104.

10. Thiering G., Londero E., Gali A. Single nickel-related defects in molecular-sized nanodiamonds for multicolor bioimaging: an ab initio study. Nanoscale 2014; 6(20): 1201812025, https://doi.org/10.1039/c4nr03112a.

11. Rabeau J.R., Chin Y.L., Prawer S., Jelezko F., Gaebel T., Wrachtrup J. Fabrication of single nickelnitrogen defects in diamond by chemical vapor deposition. Appl Phys Lett 2005; 86(13): 131926, https://doi.org/10. 1063/1.1896088.

12. Aharonovich I., Zhou C., Stacey A., Orwa J., Castelletto S., Simpson D., Greentree A.D., Treussart F., Roch J.F., Prawer S. Enhanced single-photon emission in the near infrared from a diamond color center. Phys Rev B 2009; 79(23): 235316, https://doi.org/10.1103/physrevb.79.235316.

13. Orwa J.O., Aharonovich I., Jelezko F., Balasubramanian G., Balog P., Markham M., Twitchen D.J., Greentree A.D., Prawer S. Nickel related optical centres 
in diamond created by ion implantation. J Appl Phys 2010; 107(9): 093512, https://doi.org/10.1063/1.3357374.

14. Hui Y.Y., Zhang B., Chang Y.C., Chang C.C., Chang H.C., Hsu J.H., Chang K., Chang F.H. Two-photon fluorescence correlation spectroscopy of lipid-encapsulated fluorescent nanodiamonds in living cells. Opt Express 2010; 18(6): 5896-5905, https://doi.org/10.1364/oe.18.005896.

15. Denk W., Piston D.W., Webb W.W. Multi-photon molecular excitation in laser-scanning microscopy. In: Pawley J.B. Handbook of biological confocal microscopy. Springer US; 2006; p. 535-549, https://doi.org/10.1007/978-0387-45524-2_28.

16. Nazare M.H., Neves A.J., Davies G. Optical studies of the 1.40-eV Ni center in diamond. Phys Rev B Condens Matter 1991; 43(17): 14196-14205, https://doi.org/10.1103/ physrevb.43.14196.

17. lakoubovskii K., Davies G. Vibronic effects in the 1.4-e Voptical center in diamond. Phys Rev B 2004; 70(24): 245206, https://doi.org/10.1103/physrevb.70.245206.

18. Chung P.-H., Perevedentseva E., Tu J.-S., Chang C.C., Cheng C.-L. Spectroscopic study of bio-functionalized nanodiamonds. Diam Relat Mater 2006; 15(4-8): 622-625, https://doi.org/10.1016/j.diamond.2005.11.019.

19. Davies G., Lawson S.C., Collins A.I., Mainwood A., Sharp S.J. Vacancy-related centers in diamond. Phys Rev $B$ Condens Matter 1992; 46(20): 13157-13170, https://doi. org/10.1103/physrevb.46.13157.

20. Collins A.T. The characterisation of point defects in diamond by luminescence spectroscopy. Diam Relat Mater 1992; 1(5-6): 457-469, https://doi.org/10.1016/09259635(92)90146-f.

21. Larico R., Machado W.V.M., Justo J.F., Assali L.V.C. Microscopic structure of nickel-dopant centers in diamond.
Braz J Phys 2006; 36(2a): 267-269, https://doi.org/10.1590/ s0103-97332006000300009.

22. Nadolinny V.A., Yelisseyev A.P., Yuryeva O.P., Feygelson B.N. EPR study of the transformations in nickel containing centres at heated synthetic diamonds. Appl Mag Reson 1997; 12(4): 543-554, https://doi.org/10.1007/ bf03164134.

23. Kanda H., Watanabe K. Distribution of nickel related luminescence centers in HPHT diamond. Diam Relat Mater 1999; 8(8-9): 1463-1469, https://doi.org/10.1016/s09259635(99)00070-9.

24. Zaitsev A.M. Optical properties of diamond: a data handbook. Springer-Verlag, Berlin Heidelberg; 2001, https:// doi.org/10.1007/978-3-662-04548-0.

25. Nadolinny V.A., Yelisseyev A.P., Baker J.M., Newton M.E., Twitchen D.J., Lawson S.C., Yuryeva O.P., Feigelson B.N. A study of $13 \mathrm{C}$ hyperfine structure in the EPR of nickel-nitrogencontaining centres in diamond and correlation with their optical properties. J Phys Condens Matter 1999; 11(38): 7357-7376, https://doi.org/10.1088/0953-8984/11/38/314.

26. Gucsik A., Nishido H., Ninagawa K., Ott U., Tsuchiyama A., Kayama M., Simonia I., Boudou J.-P. Cathodoluminescence microscopy and spectroscopy of microand nanodiamonds: an implication for laboratory astrophysics. Microsc Microanal 2012; 18(6): 1285-1291, https://doi. org/10.1017/s143192761201330x.

27. Collins A.T., Spear P.M. The $1.40 \mathrm{eV}$ and $2.56 \mathrm{eV}$ centres in synthetic diamond. J Phys Condens Matter 1983; 16(5): 963-973, https://doi.org/10.1088/0022-3719/16/5/023.

28. Kuo Y., Hsu T.Y., Wu Y.C., Chang H.C. Fluorescent nanodiamond as a probe for the intercellular transport of proteins in vivo. Biomaterials 2013; 34(33): 8352-8360, https:// doi.org/10.1016/j.biomaterials.2013.07.043. 